\title{
Analytical Deflection Profiles and Pull-In Voltage Calculations of Prestressed Electrostatic Actuated MEMS Structures
}

\section{Havreland, Andreas Spandet; Thomsen, Erik Vilain}

Published in:

I E E E Journal of Microelectromechanical Systems

Link to article, DOI:

10.1109/JMEMS.2021.3083935

Publication date:

2021

Document Version

Peer reviewed version

Link back to DTU Orbit

Citation (APA):

Havreland, A. S., \& Thomsen, E. V. (2021). Analytical Deflection Profiles and Pull-In Voltage Calculations of Prestressed Electrostatic Actuated MEMS Structures. I E E E Journal of Microelectromechanical Systems, 30(4), 659 - 667. https://doi.org/10.1109/JMEMS.2021.3083935

\section{General rights}

Copyright and moral rights for the publications made accessible in the public portal are retained by the authors and/or other copyright owners and it is a condition of accessing publications that users recognise and abide by the legal requirements associated with these rights.

- Users may download and print one copy of any publication from the public portal for the purpose of private study or research.

- You may not further distribute the material or use it for any profit-making activity or commercial gain

- You may freely distribute the URL identifying the publication in the public portal 


\title{
Analytical Deflection Profiles and Pull-In Voltage Calculations of Prestressed Electrostatic Actuated MEMS Structures
}

\author{
Andreas Spandet Havreland ${ }^{\circledR}$ and Erik Vilain Thomsen
}

\begin{abstract}
This paper presents a zeroth and first order perturbative analysis of prestressed electrostatic actuated Microelectromechanical systems (MEMS). Perturbation theory is used to calculate the deflection profile of various MEMS structures, from which the pull-in voltage is estimated using the weighed residual method, where both a Galerkin expression and a Dirac delta function have been used as weight functions. A prestressed circular Capacitative Micromachined Ultrasonic Transducer (CMUT) is used as the main example in this paper. This device is modeled as a circular clamped plate subjected to an electrostatic pressure. The calculated pull-in voltage has been compared with experimental data of highly prestressed CMUTs, where a relative error between $-36 \%$ and $-\mathbf{8 \%} \%$ is observed for a model that does not include stress. The model that includes the residual stress lowers the range of the relative error to values between $-5 \%$ and $21 \%$. To improve the accuracy of the pull-in voltage estimate Richardson extrapolation has been calculated from the zeroth and first order estimates. The pull-in voltage models are compared with a Finite Element Model (FEM), where an overestimation, in the high stress regime, of $10 \%$ is observed for the zeroth order model, less than $\mathbf{5 \%}$ for the Galerkin method and $3 \%$ for the Richardson extrapolation.

[2020-0358]
\end{abstract}

Index Terms-Micromechanical devices, nonlinear equations, capacitive transducers.

\section{INTRODUCTION}

$\mathbf{T}$ HE electrostatic principle has been used to mechanically manipulate Micro Electrical Mechanical Systems (MEMS) for more than two decades. The applications of electrostatically actuated MEMS devices include among others RF switches [1], micro-mirrors [2], comb-drives [3], chemical sensing [4], and ultrasound transducers [5]. The electrostatic force couples the electrical and mechanical domains, and this coupling increases in a non-linear manner as the applied voltage increases. MEMS devices are known for high coupling coefficients, and experimental coupling coefficients as high as 0.85 have been reported for electrostatic devices [6]. However, the advantageous non-linear electrostatic coupling does introduce a saddle-node bifurcation known as pull-in, where the movable part collapses into the static part of the

Manuscript received October 27, 2020; revised May 3, 2021; accepted May 20, 2021. This work was supported in part by the Danish Innovation Fund and in part by BK Medical. Subject Editor N. Barniol. (Corresponding author: Andreas Spandet Havreland.)

The authors are with the Department of Health Technology, Technical University of Denmark, DK-2800 Kongens Lyngby, Denmark (e-mail: ahav@nanotech.dtu.dk).

Color versions of one or more figures in this article are available at https://doi.org/10.1109/JMEMS.2021.3083935.

Digital Object Identifier 10.1109/JMEMS.2021.3083935 device when the applied voltage is larger than the so-called pull-in voltage. Knowing the pull-in voltage is crucial for correct operation of CMUTs, and the understanding of this phenomenon is thus highly important prior to the design and fabrication phase. CMUTs are commonly operated below the pull-in voltage to reduce effects from mobile charges, but stable CMUTs operated above the pull-in voltage have been demonstrated [7]-[9].

Various models for pull-in instabilities have been demonstrated in the literature [10]-[16], but these models do commonly not include the effect from a built-in biaxial stress. Some of the published models include an analytical pull-in model for different geometries presented by Osterberg [17], where numerical constants are found from fits to calculations from a finite element method (FEM). A Galerkin approach has been used by Nayfeh to calculate various parameters including the pull-in voltage for both a clamped beam and a clamped plate in [18] and [19], respectively. This paper presents a general methodology for estimations of the pull-in voltage of various MEMS devices, and this method allows for simple closed-form expressions and valuable insight about the scaling properties at different orders of approximation as well as easy implementation of arbitrary forces. Commonly, the static deflection is used as a trail function, however, the presented methodology utilizes a perturbative approach which includes the effect of a non constant electrostatic pressure.

The focus of this paper is MEMS devices with a significant biaxial stress in the movable MEMS part, however, the analysis can also be conducted when other forces are present or when there are a combination of multiple forces. The Capacitative Micromachined Ultrasonic Transducer (CMUT) will be analyzed as the MEMS device in this paper. The CMUT was first proposed by Haller and Khuri-Yakub back in the 1990's [20], and has since then primarily been used for medical imaging [5] and sensing applications [4]. A CMUT consists of a rigid substrate, a cavity, and a movable plate as illustrated in Fig. 1. An electric insulator separates the plate and the substrate, and actuation of the CMUT occurs when a potential is applied between plate and the substrate, which in the electrical domain functions as electrodes. The geometry of a CMUT is defined in a lithography process, where circular or square geometries typically are used. Multiple circular CMUTs can be packed tightly together in a hexagonal grid, which is the typical approach when designing CMUT arrays. In addition, the circular geometry, and thus the use of polar coordinates, 
Movable plate/top electrode

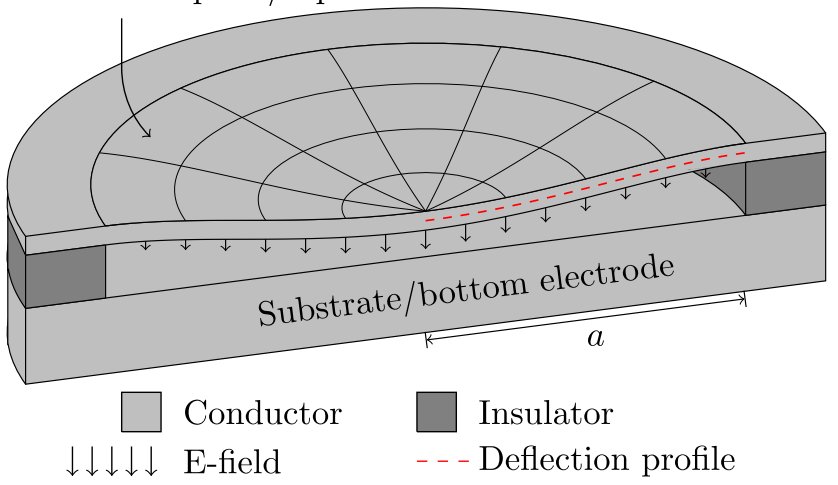

Fig. 1. Sketch of a clamped circular plate with radius $a$. The dashed red line is the deflection profile that is used to estimate the pull-in voltage. The arrows indicate the E-field, which is largest at the center due to the reduced gap height.

does also offer closed-form solutions to the linear plate equation, opposite to the use of Cartesian coordinates where exact solutions have to be expressed in terms of an infinite series.

This paper is organized as follows: The methodology of a general pull-in model for electrostatic MEMS devices is given in section II. Estimates of the pull-in voltage and the deflection profile are then calculated for four different geometries and boundary conditions. The developed pull-in models are compared with FEM simulations and experimental data in section III, where three CMUTs with a significant built-in stress are used as the experimental reference. Finally, section IV concludes the paper.

\section{THEORY}

The deflection of an isotropic plate is governed by

$$
D \nabla^{2} \nabla^{2} w-\sigma_{0} h \nabla^{2} w=p,
$$

here expressed in a coordinate free form [21]. $w$ is the deflection profile, $D$ is the flexural rigidity, $\sigma_{0}$ is the biaxial stress, $h$ is the plate thickness, and $p$ is the applied pressure. The flexural rigidity is given by

$$
D=\frac{E h^{3}}{12\left(1-v^{2}\right)},
$$

where $E$ is Young's modulus and $v$ is the Poisson's ratio. For an electrostatically controlled MEMS structure, the electrostatic pressure is given by

$$
p=\frac{\varepsilon_{0} V^{2}}{2 g^{2}(1-w / g)^{2}},
$$

where $\varepsilon_{0}$ is the vacuum permittivity, $V$ is the applied voltage, and $g$ is the initial gap height. The operators used in Eqn. (1) are provided for Cartesian and polar coordinates in Appendix A. Eqn. (1) is valid under the assumption of linear strain and isotropic material properties. The typical lateral dimension of an electrostatic MEMS structure is much larger than the distance between the electrodes, and effects from the fringing fields and the bending of the electric field lines inside the cavity are therefore small. The fringing fields will begin to
TABLE I

NormaLized PARAMETERS

\begin{tabular}{lll}
\hline Parameter & Definition & Typical value range \\
\hline$\hat{w}$ & $\frac{w}{g}$ & {$[0-1]$} \\
\hline$\hat{r}$ & $\frac{r}{a}$ & {$[0-1]$} \\
\hline$\hat{\sigma}$ & $\frac{\sigma_{0} h a^{2}}{D}$ & {$[0-500[$} \\
\hline$\hat{\alpha}$ & $\frac{\varepsilon_{0} V^{2} a^{4}}{2 D g^{3}}$ & {$[0-17[$} \\
\hline
\end{tabular}

make an impact when the aspect ratio of the CMUT plate is decreased [22], however, most CMUT applications are in the high aspect ratio regime where the impact from the fringing field is small. In the case where the plate is displaced more than two times the plate thickness the fringing fields will also influence the pull-in voltage, as shown in [23] where $11 \%$ relative difference is observed between the models with and without fringing fields.

The dominating force that pulls the plate towards the substrate is assumed to be the electrostatic force, however, for small the gap heights, other forces such as the Van der Wall force [24] and the Casimir force become significant [25].

This work investigates a single circular CMUT cell with radius $a$. The boundary is fully clamped, although, a real device will have a small elasticity at the boundary [26]. Altered boundary conditions due to array effects [27] are not included in the developed model.

A non-linear differential equation emerges when the electrostatic pressure, Eqn. (3), is substituted into Eqn. (1). If all terms are moved to the left hand side and dimensionless parameters are introduced the non-linear differential equation can be expressed as

$$
(1-\hat{w})^{2}\left(\nabla^{2} \nabla^{2} \hat{w}-\hat{\sigma} \nabla^{2} \hat{w}\right)-\hat{\alpha}=0,
$$

where all dimensionless parameters are indicated using a hat notation. The dimensionless parameters are defined in Table I. Notice, the dimensionless stress parameter, $\hat{\sigma}$, is highly sensitive to the size of the CMUT cell due to the square dependence on the plate aspect ratio, $a / h$, which is seen if the flexural rigidity, Eqn. 2 , is inserted into the expression for $\hat{\sigma}$, which then yields

$$
\hat{\sigma}=\frac{\sigma_{0} h a^{2}}{D}=\frac{12 \sigma_{0}\left(1-v^{2}\right)}{E}\left(\frac{a}{h}\right)^{2} .
$$

An exact solution to Eqn. (4) is not known and an approximate trial function, $\hat{w}_{a}$, must therefore be used instead, which introduces a residual term, $\hat{R}$, given by

$$
\left(1-\hat{w}_{a}\right)^{2}\left(\nabla^{2} \nabla^{2} \hat{w}_{a}-\hat{\sigma} \nabla^{2} \hat{w}_{a}\right)-\hat{\alpha}=\hat{R} .
$$

Minimization of $\hat{R}$ can be done by the method of weighted residuals, which has been employed in this work. Various weighting functions can be chosen and two different weight functions are evaluated. A Dirac delta function is used to obtain compact simple closed-form pull-in expressions which are easy to interpret. The widely accepted Galerkin method 
is used as the second weight function with the purpose of validating the proposed methodology. The trial function, $\hat{w}_{a}$, must satisfy the imposed boundary conditions at $\hat{r}=0$ and $\hat{r}=1$, this causes the residual error to be zero at the boundaries. The Dirac delta function introduces an additional point with zero error, and to evenly distribute the points of zero error the weight is chosen to be at half radius, $\hat{r}=1 / 2$, which reduces the minimization problem to

$$
\int_{0}^{1} \delta\left(\hat{r}-\frac{1}{2}\right) \hat{R} \mathrm{~d} \hat{r}=0
$$

The Galerkin method uses orthogonal trial functions to estimate solutions to differential equations. The static deflection profile is in our work used as the trial function for the Galerkin method. In terms of simplicity the Galerkin method has the drawback of producing expressions with multiple $\hat{\sigma}$ terms at different orders, typically on the order of ten terms in this type of analysis. The Galerkin solution has for completeness been calculated analytically and can be found in Appendix B. The Dirac delta weight function yields, on the other hand, a pullin expression with only two terms, a term that describes the scaling of the stress free plate and an addition stress correction term.

The presented equations are up until this point generic for any given plate geometry. However, the deflection profile of the plate is required to proceed with the subsequent steps. Other geometries will be discussed in section II-B, but henceforth the assumed geometry is a circular plate as shown in Fig. 1. The magnitude of the electrostatic pressure is shown by the arrows below the plate, and the red dashed line illustrates the deflection profile, which has to be approximated by the trial function. The trial function used in this analysis is the static deflection of a stress free clamped plate subjected to a uniform applied pressure, which is given by [21]

$$
\hat{w}(\hat{r})=\hat{A}\left(1-\hat{r}^{2}\right)^{2},
$$

where $\hat{A}$ is an amplitude parameter and $\hat{r}$ is the normalized radius. The plate in this analysis is subjected to the following boundary conditions

$$
\hat{w}(0) \rightarrow \text { finite, } \hat{w}^{\prime}(0)=0, \quad \hat{w}(1)=0, \hat{w}^{\prime}(1)=0 .
$$

Eqn. (8) is exact when a uniform pressure is applied, but only an approximate solution when the plate is subjected to an electrostatic pressure. Hence, the final estimate of the pull-in voltage will therefore also be an approximation.

To find the specific amplitude, $\hat{A}$, at which the pull-in phenomenon occurs the approximate deflection profile Eqn. (8) is substituted into Eqn. (7) which then yields

$$
\left(1-\frac{9 \hat{A}}{16}\right)^{2}(64 \hat{A}+4 \hat{A} \hat{\sigma})-\hat{\alpha}=0 .
$$

This equation is cubic in $\hat{A}$, and $\hat{\alpha}$ is a variable that varies with the applied voltage, whereas the parameter $\hat{\sigma}$ will be constant for a given CMUT design. Stable solutions to Eqn. (4) can be obtained as long as $\alpha$ is below a certain threshold. This threshold occurs when the discriminant of the third order polynomial equals zero, which will be the last (semi-)stable state before the pull-in point.

A generic third order polynomial and its corresponding discriminant, $\Delta$, are given by

$$
c_{3} x^{3}+c_{2} x^{2}+c_{1} x+c_{0}=0,
$$

and

$$
\Delta=c_{2}^{2} c_{1}^{2}-4 c_{3} c_{1}^{3}-4 c_{2}^{3} c_{0}-27 c_{3}^{2} c_{0}^{2}+18 c_{3} c_{2} c_{1} c_{0} .
$$

The discriminant of Eqn. (10) equals zero when

$$
\frac{4096}{243}\left(1+\frac{\hat{\sigma}}{16}\right)=\hat{\alpha}_{p i}=\frac{\varepsilon_{0} V_{p i}^{2} a^{4}}{2 D g^{3}} \text {. }
$$

Thus, by rearranging Eqn. (13) the zeroth order pull-in voltage can be found by

$$
V_{p i}^{(0)}=\frac{64}{9} \sqrt{\frac{2}{3} \frac{D g^{3}}{\varepsilon_{0} a^{4}}\left(1+\frac{\hat{\sigma}}{16}\right)} .
$$

The superscript ${ }^{(0)}$ is introduced to show the expansion order of the electrostatic pressure, in this case the zeroth order. The term $\hat{\sigma} / 16$ inside the parenthesis of Eqn. (14) can be perceived as a correction term to the stress free state. As a sanity check the scaling properties obtained in this expression are identical to previously reported pull-in models in the literature [11], [12], [14].

The importance of the built-in stress is determined by the dimensionless stress factor $\hat{\sigma}$, and a high tensile residual stress increases the pull-in voltage as expected. Furthermore, this expression predicts that buckling will occur when $\hat{\sigma}<-16$, in accordance with [28].

The increased pull-in voltage for tensile plates can be visualized by the potential energy experienced by the CMUT, which can be found by integrating the electrostatic plate equation, Eqn 1, yielding

$$
\hat{U}=\int \nabla^{2} \nabla^{2} \hat{w} \mathrm{~d} \hat{A}-\hat{\sigma} \int \nabla^{2} \hat{w} \mathrm{~d} \hat{A}-\int \frac{\hat{\alpha}}{(1-\hat{w})^{2}} \mathrm{~d} \hat{A} .
$$

If a static zeroth order solution is used as trial function and evaluated at $\hat{r}=1 / 2$ one gets

$$
\hat{U}^{(0)}=32 \hat{A}^{2}+2 \hat{A}^{2} \hat{\sigma}-\frac{16}{9} \frac{\hat{\alpha}}{1-\frac{9 \hat{A}}{16}}
$$

where $\hat{U}$ is the normalized potential energy per area. The potential energy increases linearly with $\hat{\sigma}$, whereas the electrostatic term lowers the potential energy for all values of $\hat{\alpha}$. The effect from the built-in stress is shown in Fig. 2 as a change in the potential energy.

The solid lines correspond to a stress free plate, and the dashed lines correspond to a plate where $\hat{\sigma}=2$. The stationary point shifts to a lower displacement value when the plate has a tensile stress and more energy is stored when the bias is increased as one would expect. In addition, the curvature of the stressed energy potential is higher than the stress free, meaning the plate will move less whenever the plate is actuated by an external force.

The experienced potential energy is not uniform along the deflection profile. The center point will be closest point to 


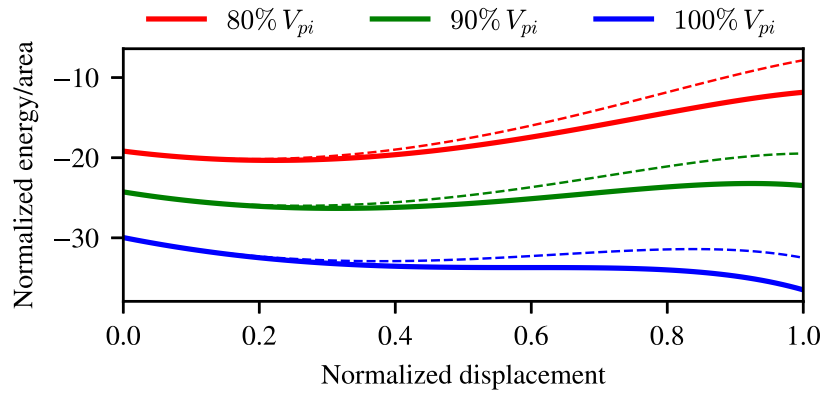

Fig. 2. Energy landscape for a CMUT at $80 \%, 90 \%$, and $100 \%$ of the pullin voltage. The bias is here referenced for a plate without stress. The solid lines correspond to a stress free plate, whereas the dashed lines correspond to a plate where $\hat{\sigma}=2$. These energy potentials are calculated for the point located at $\hat{r}=1 / 2$.

the bottom of the cavity and therefore experience the largest electrostatic force. The point at the deflection profile located at half of the radius, $\hat{r}=1 / 2$, will therefore experience a reduced electrostatic force. This is the reason why the potential energy does not diverge to minus infinity as the normalized distance approaches one.

The methodology is not restricted to built-in stress, but can also be applied for other force contributions such as an external pressure difference across the CMUT plate. A calculation of the pull-in voltage with built-in stress and an external pressure difference can be found in Appendix C.

\section{A. First Order Correction}

The electrostatic pressure depends on the distance, $g-w(r)$, between the top and bottom electrode. To account for this effect the electrostatic pressure is expanded

$$
\begin{aligned}
\hat{p} & =\frac{\hat{\alpha}}{(1-\hat{w})^{2}} \\
\hat{p}^{(0)} & =\hat{\alpha} \\
\hat{p}^{(1)} & =\hat{\alpha}(1+2 \hat{w}) .
\end{aligned}
$$

Hence, to first order the plate equation can be written as

$$
\nabla^{2} \nabla^{2} \hat{w}-\hat{\sigma} \nabla^{2} \hat{w}=\hat{\alpha}(1+2 \hat{w}),
$$

once again expressed by dimensionless parameters. An exact solution to this equation can be found by mathematical software programs such as Mathematica and Maple, and the solution is composed of multiple Bessel functions both modified and first kind. Although this represents the exact solution, it can be difficult to interpret and not easy to implement in the presented methodology. Alternatively, one can find highly accurate approximate solutions in terms of polynomials. If $\hat{\sigma}$ and $2 \hat{w}$ are assumed small, Eqn. (20) reduces to the unstressed plate equation with a uniform pressure, where the solution is known exactly and given by

$$
\hat{w}^{(0)}=\frac{\hat{\alpha}\left(1-r^{2}\right)^{2}}{64} .
$$

Thus, the trial function used is the solution to the unperturbed system, and a correction is found by introducing $\hat{\sigma}$ and $2 \hat{w}$ to first order. The solution is the sum of the unperturbed solution, $\hat{w}^{(0)}$, and the first order correction, $\hat{w}^{(1)}$, given by

$$
\hat{w} \approx \hat{w}^{(0)}+\hat{w}^{(1)} .
$$

The first order solution is found by the coupled equations

$$
\begin{aligned}
& \nabla^{2} \nabla^{2} \hat{w}^{(0)}=\hat{\alpha} \\
& \nabla^{2} \nabla^{2} \hat{w}^{(1)}=2 \hat{\alpha} \hat{w}^{(0)},
\end{aligned}
$$

which are solved iteratively yielding the solution

$$
\hat{w} \approx \hat{w}^{(0)}\left(1+\frac{23-6 r^{2}+r^{4}}{1152}\right) .
$$

This first order correction introduces a slight change to the deflection profile and adds additional displacement at the center due to the larger electrostatic pressure at this position. If the first order correction, Eqn. (25), is used as the trial function the estimated pull-in voltage becomes

$$
V_{p i}^{(1)}=\frac{128}{3} \sqrt{\frac{2}{115} \frac{D g^{3}}{\varepsilon_{0} a^{4}}\left(1+\frac{\hat{\sigma}}{16}\right)} .
$$

Notice, the first order correction lowers the magnitude of the estimated pull-in voltage by a factor of $\sqrt{108 / 115} \approx 0.97$, compared to Eqn. (14). A comparison between the exact solution to Eqn. (20) and the approximate polynomial solution, Eqn. (25), yields a maximum relative error less than $0.04 \%$ when the calculated pull-in voltage is used.

The expansion of the electrostatic pressure, Eqn. (3), consists exclusively of positive terms, and it translates into an ever increasing pressure whenever the order of the series is increased. The estimation of the pull-in voltage will therefore approach its limit monotonically as the expansion order is increased. The accuracy of the pull-in voltage expression improves as the perturbation order is increased, however, going from first order to higher order corrections can be cumbersome. Therefore, an accelerated convergence rate is an appealing alternative. The accuracy can in this case be improved using Richardson extrapolation [29], a method that accelerates the convergence rate of a sum that approaches its limit monotonically. In this case where the zeroth and first order estimates are known, the lowest order Richardson extrapolation is given by

$$
\begin{aligned}
V_{p i}^{(R)} & =-V_{p i}^{(0)}+2 V_{p i}^{(1)} \\
V_{p i}^{(R)} & =\frac{64(36 \sqrt{230}-115 \sqrt{6})}{3105} \sqrt{\frac{D g^{3}}{\varepsilon_{0} a^{4}}\left(1+\frac{\hat{\sigma}}{16}\right)}
\end{aligned}
$$

Here the superscript $(R)$ indicates the Richardson extrapolation. This is similar to Eqn. (26) but has slightly different constants in the expression.

\section{B. Other Geometries}

The presented methodology is not restricted to circular plates, but can be applied to many other geometries as well. This is illustrated in Table II, where deflection profiles and pull-in voltages are calculated for a clamped square plate, a clamped beam, and a cantilever beam. The pull-in voltage 
TABLE II

Calculated Deflection Profiles and Pull-in Voltages for Other Geometries, and the Boundary Conditions (B.C) Used

\begin{tabular}{llll}
\hline \multicolumn{1}{c}{ Clamped Square plate } & Clamped beam & Cantilever beam \\
\hline$\hat{w}^{(0)}$ & $A(x-1)^{2}(y-1)^{2}$ & $A x^{2}(x-1)^{2}$ & $A x^{2}\left(x^{2}-4 x+6\right)$ \\
$\hat{w}^{(1)}$ & - & $\hat{w}^{(0)}\left(\frac{9+4 x-x^{2}-6 x^{3}+3 x^{4}}{2520}\right)$ & $\hat{w}^{(0)}\left(\frac{728-336 x+24 x^{4}-8 x^{5}+x^{6}}{35\left(6-4 x+x^{2}\right)}\right)$ \\
$V_{p i}^{(0)}$ & $\frac{32}{27} \sqrt{\frac{58}{3} \frac{D g^{3}}{\varepsilon_{0} a^{4}}\left(1+\frac{9}{232} \hat{\sigma}\right)}$ & $\frac{32}{3} \sqrt{\frac{E I g^{3}}{\varepsilon_{0} W L^{4}}\left(1+\frac{\hat{N}}{24}\right)}$ & $\frac{16}{3} \sqrt{\frac{1}{51} \frac{E I g^{3}}{\varepsilon_{0} W L^{4}}\left(1-\frac{\hat{N}}{8}\right)}$ \\
$V_{p i}^{(1)}$ & - & $32 \sqrt{\frac{70}{489} \frac{E I g^{3}}{\varepsilon_{0} W L^{4}}\left(1+\frac{29 \hat{N}}{840}\right)}$ & $\frac{32}{99} \sqrt{\frac{1190}{33} \frac{E I g^{3}}{\varepsilon_{0} W L^{4}}\left(1-\frac{11 \hat{N}}{40}\right)}$ \\
\hline B.C & $w( \pm 1, \pm 1)=w^{\prime}( \pm 1, \pm 1)=0$ & $w(0)=w^{\prime}(0)=w(1)=w^{\prime}(1)=0$ & $w(0)=w^{\prime}(0)=w^{\prime \prime}(1)=w^{\prime \prime \prime}(1)=0$
\end{tabular}

of the square plate is calculated using Eqn. (1) in Cartesian coordinates, however, in this case the zeroth order deflection cannot be expressed in a simple closed-form, and an approximate solution is therefore used instead. Since the zeroth order solution is approximate the calculations of the first order solution is omitted. An exact solution can be expressed as an infinite series, but, such solution is not well suited for the presented methodology. A series solution model for the stressed clamped rectangular plate is derived in [30], and a description of layered and stressed plates can be found in [28], [31].

The Euler-Bernoulli beam equation subjected to an electrostatic load is used for the clamped beam and the cantilever beam and formulated as

$$
E I \frac{\mathrm{d}^{4} w}{\mathrm{~d} x^{4}}-N \frac{\mathrm{d}^{2} w}{\mathrm{~d} x^{2}}=q
$$

where $N$ is the tension and $q$ is the electrostatic load that can be expressed as

$$
q=\frac{\varepsilon_{0} W V^{2}}{2 g^{2}(1-w / g)}
$$

where $W$ is the width of the beam and $I$ is the area moment of inertia, which for a rectangular beam is defined as

$$
I=\frac{W h^{3}}{12} .
$$

The boundary conditions used are provided mathematically in the bottom of Table II, and sketches of the geometries can be found in Fig. 3. The dimensionless versions of the EulerBernoulli equation can be found in Appendix D.

The material and geometry scaling properties given in Table II are identical to the theory presented in [17], as one would expect.

\section{RESULTS AND DISCUSSION}

The derived pull-in models have been compared with both FEM simulations and experiential data [32]-[34]. The accuracy of the models are investigated using FEM simulation and thus under a fully controlled environment. The FEM model is made in COMSOL version 5.5. Deformation in the mechanical domain is calculated using the Solid Mechanics module and the changes in the electrical domain are calculated
TABLE III

FEM PARAMETERS

\begin{tabular}{lll}
\hline Simulation & A & B \\
\hline Plate radius & $15 \mu \mathrm{m}$ & $15 \mu \mathrm{m}$ \\
Young's modulus & $170 \mathrm{GPa}$ & $170 \mathrm{GPa}$ \\
Poisson's ratio & 0.28 & 0.28 \\
\hline Plate thickness & $300 \mathrm{~nm}$ & $400 \mathrm{~nm}$ \\
Gap height & $400 \mathrm{~nm}$ & $100 \mathrm{~nm}$ \\
\hline
\end{tabular}

by the Electrostatic module. The two domains are coupled by a Moving Mesh and the Multiphysics module Electromechanical Forces. The pull-in voltage is determined by the Optimization module using the Nelder-Mead optimization solver.

Two sets of CMUT parameters have been used. The first set of parameters is denoted simulation A, and these parameters are similar to the design parameter used for the three evaluated CMUTs in [32]-[34]. The parameters used for the second simulation, $\mathrm{B}$, are chosen such that the deflection of the plate is small compared to the plate thickness, or in other words in the linear elastic regime. The built-in stress, $\sigma_{0}$, is swept from -90 MPa to $1300 \mathrm{MPa}$. Stress values within this span can be achieved experimentally and do not reach the buckling regime for the chosen CMUT parameter provided in Table III for both simulation A and B.

The calculated pull-in voltage as function of $\hat{\sigma}$ is plotted in Fig. 4 for both simulation A and B. The numerical results from COMSOL is shown as black dots, and the zeroth order model (Eqn. 14) is plotted using a solid line, the Richardson extrapolation (Eqn. 28) is plotted using a dotted line, and finally the Galerkin solution is plotted using a dashed line. The red lines illustrate the relative error on the right y-axis. The zeroth order solution deviates up to $10 \%$ from the FEM data in the tensile regime, and deviates more than the Richardson and the Galerkin solution as one would expect. The Richardson extrapolation and the Galerkin solution have similar precision across the tensile regime. The Richardson extrapolation is, however, slightly better in the highly tensile regime $(\hat{\sigma} \gg 1)$ with a maximum relative error less than $3 \%$ for both simulation $\mathrm{A}$ and $\mathrm{B}$, whereas the error is less than $5 \%$ for the Galerkin solution. The Richardson extrapolation and the Galerkin solution both have a precision better than 5\% in the entire tensile stress range $(\hat{\sigma} \gg 1)$, whereas the zeroth order model is accurate within $10 \%$ in the same regime. The models 


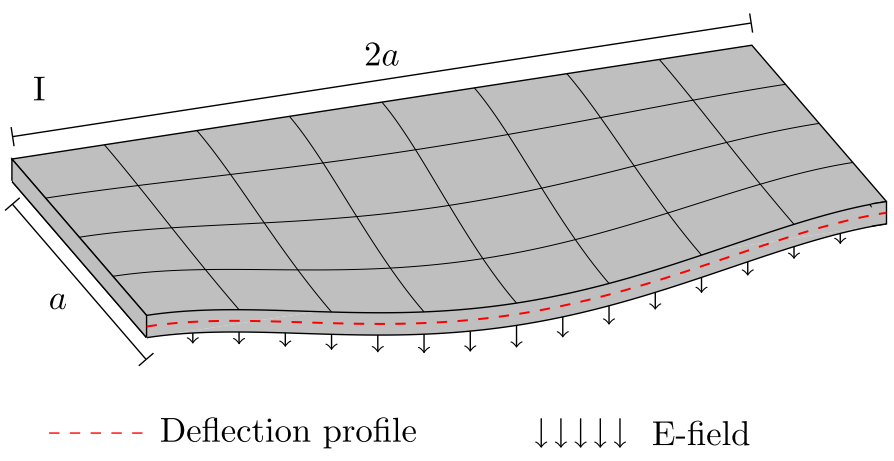

Fig. 3. Sketches of the geometries used in Table II. I) is the clamped square plate, notice only half of the geometry is drawn in order to visualize the deflection profile. II) is the clamped beam, and III) is the cantilever beam. Relevant geometrical parameters are included in each sub-figure.

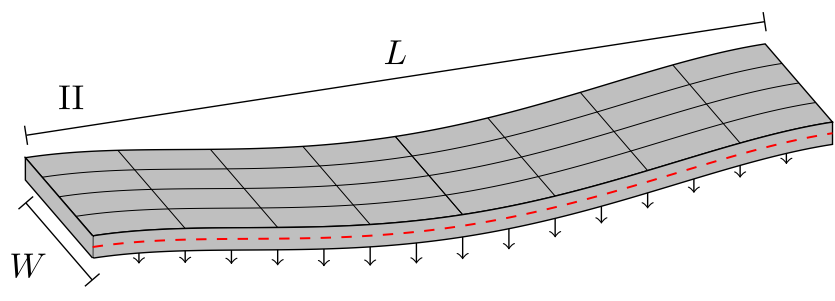

III $L$

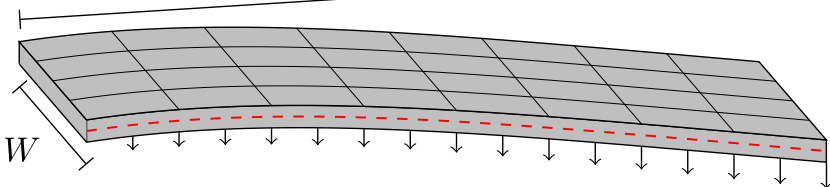

TABLE IV
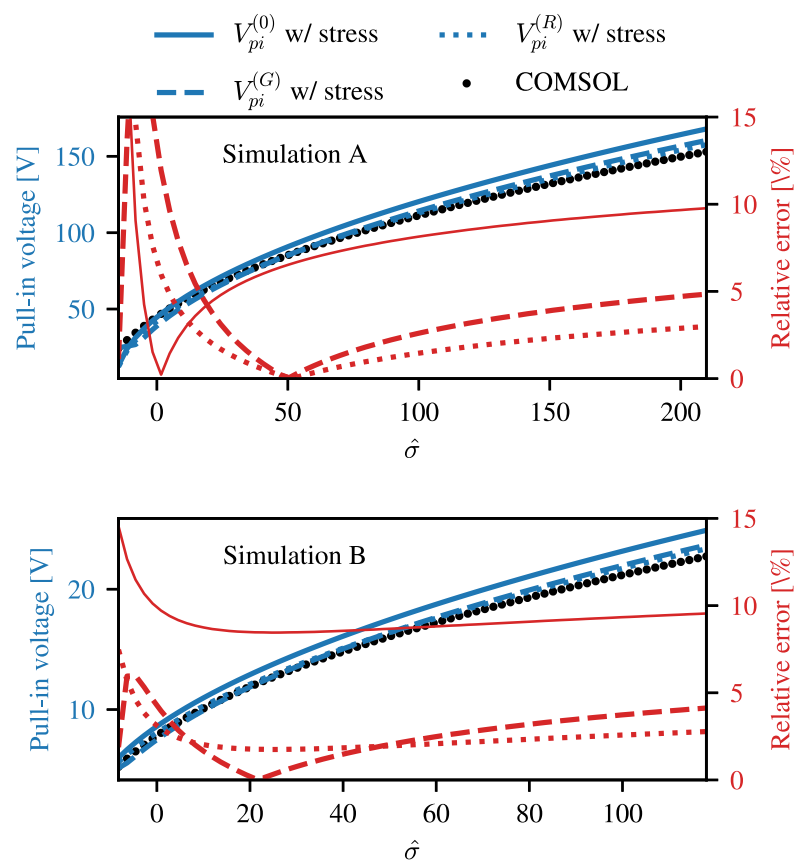

Fig. 4. The developed pull-in models validated against a COMSOL simulation. The result from the FEM simulation is indicated with the black dots. The blue curves represent the pull-in estimate of the various models, and the red curves show the relative error. Notice, the range of both the $x$ and $y$ axes differ between the two plots.

underestimate the pull-in voltage in the compressive and near stress-free regimes in simulation A, whereas the models overestimates the pull-in voltage in the highly tensile stress regime. The COMSOL simulation includes other nonlinear effects such as higher order strain relations, which are not included in the analytical model. Thus, higher order strains become significant for large plate displacement, relative to the plate thickness, which is the case for simulation A. Despite a linear displacement assumption the estimated pull-in voltage is still accurate within $10 \%$ for all models as long as the built-in stress is tensile.
PARAMETERS

\begin{tabular}{llll}
\hline Ref & {$[32]$} & {$[33]$} & {$[34]$} \\
\hline Plate material & $\mathrm{Si}_{3} \mathrm{~N}_{4}$ & $\mathrm{Si}_{3} \mathrm{~N}_{4}$ & $\mathrm{Si}_{3} \mathrm{~N}_{4}$ \\
Plate thickness [nm] & 350 & 400 & 100 \\
Plate radius [ $\mu \mathrm{m}]$ & 30 & 11 & 5 \\
Gap height [nm] & 450 & 140 & 255 \\
Biaxial stress [MPa] & $300^{a}$ & 200 & 1300 \\
Top electrode thickness [nm] & 400 & 130 & 100 \\
Pull-in voltage [V] & 75 & 55 & 150 \\
Structured top electrode & $\mathrm{No}$ & Yes & Yes \\
\hline$\hat{\sigma}$ & 147 & 10.2 & 220 \\
$D_{\text {eff }} / D$ & 6.16 & 1.65 & 5.02 \\
$\hat{\sigma}_{\text {eff }} / \hat{\sigma}$ & 0.16 & 0.60 & 0.20 \\
\hline & & &
\end{tabular}

The gap is lowered and the plate thickness is increased in Simulation B to reduce effects from nonlinear strain. The models overestimate the pull-in voltage for all values of $\hat{\sigma}$, and the accuracy increases between the zeroth order and the Richardson expression as expected.

Tensile stress is preferred over compressive stress for CMUT applications, hence, the regime of interest is $\hat{\sigma} \gg 1$.

The developed pull-in models are validated against experimental values found in the literature for three different CMUTs [32]-[34]. The relevant CMUT parameters are provided in Table IV for the three CMUTs, all of which are fabricated using a silicon nitride plate with tensile stress. The magnitude of the calculated dimensionless stress parameter, $\hat{\sigma}$, can be found in Table IV. The CMUTs do all have a layer of aluminum as top electrode and the mechanical influence from having a multi layered plate is implemented using an effective flexural rigidity, $D_{\text {eff }}$, [35] and an effective built in stress, $\hat{\sigma}_{\text {eff }},[28]$. The top electrode covers the CMUT entirely in [32] and the assumption for the used multi layered plate theory is therefore met. However, the plate is only partially covered with aluminium in [33] and [34], since the top electrode is structured to lower the parasitic capacitance. The multi layered plate theory will therefore overestimate the stiffness of the plate and therefore also the pull-in voltage. The input parameters for the aluminum top electrode is a Young's

\footnotetext{
${ }^{1}$ Value not reported in paper, but found in our process logbook.
} 


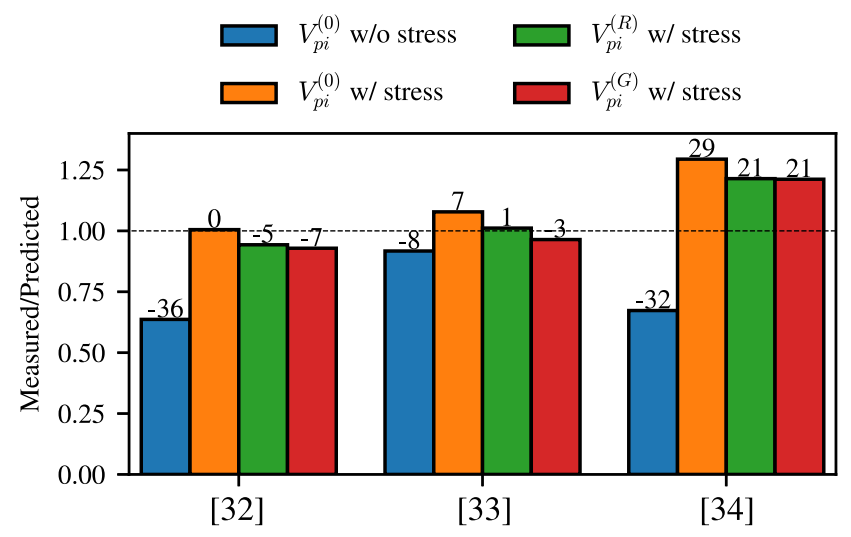

Fig. 5. The pull-in estimations of the developed models plotted against experimental values from the literature. The dashed line corresponds to the measured value and it has been normalized to the predicted values from the developed models. The relative percentage error is stated at the top of each bar. The inclusion of the stress reduces the relative error in all three cases. The zeroth order pull-in voltage is calculated using Eqn. (14), the Richardson pull-in voltage is calculated using Eqn. (28), and the pull-in voltage from a Galerkin approach, $V_{p i}^{(G)}$, is included as a reference methodology.

modulus of $70 \mathrm{GPa}$, a Poisson's ratio of 0.3 , and zero builtin stress. The ratio of the new effective properties are listed in Table IV relative to the parameters without the aluminium top electrode.

The pull-in voltage estimations with and without built-in stress are compared in Fig. 5. The relative error is stated at the top of each bar, and ranges from $-36 \%$ to $-8 \%$ for the model without stress, whereas the relative error is between $1 \%$ to $21 \%$ for the Richardson model where the built-in stress is included. Hence, a significant improvement in accuracy is observed for the stress models.

The assumption for the multi layered plate theory is fully met in [32], and the pull-in estimation is also in very good agreement with the experimental result. The top electrode is structured in both [33] and [34], and the assumption for the multi layered plate theory is therefore not fully met, which can explain the deviation between our model and the experimental data from [34]. The effective flexural rigidity is 5.02 in [34], and the effect from the aluminum is therefore much more pronounced in [34] compared to [33] where the effective flexural rigidity is 1.65 . Thus, the overestimation is expected to be larger for the device presented in [34], which is in agreement with the observation.

The structured aluminum electrode is one error source, another pronounced error source is the uncertainties on the input parameters, in particular the thin film stress is difficult to determine with high accuracy and precision, and errors on the order of hundred $\mathrm{MPa}$ is not uncommon. Uncertainties associated with the plate thickness and gap height are also significant as they are raised to the $3 / 2^{\text {th }}$ power in the pull-in expression.

\section{CONCLUSiON}

A methodology for pull-in estimation of various prestressed electrostatically actuated MEMS structures was presented.
Both zeroth and first order deflection profiles were calculated and used for a zeroth and first order estimation of the pull-in voltage. The presented methodology was based on weighted residual, and a Dirac delta weight was used to obtain simple closed-form expressions, whereas the well-known Galerkin weight was used as a validating reference. Experimental pullin voltages for circular CMUTs were compared with the developed pull-in models. The relative error ranged between $-36 \%$ and $-8 \%$ when stress was not accounted for in the pull-in model, whereas the relative error was lowered to range between $21 \%$ and $-5 \%$ when stress was included in the model. In addition, the developed pull-in models were validated by a finite element model in COMSOL, where the pull-in voltage was calculated as function of built-in stress in the plate. Excellent agreement between the developed analytical models and results from COMSOL was observed for large tensile stresses, where the zeroth order model overestimated the pull-in voltage with less than $10 \%$, the Galerkin solution overestimated with less than $5 \%$, and the Richardson model overestimated the numerical value from COMSOL with only $3 \%$.

\section{APPENDIX A}

The biharmonic operator is given by

\section{A. Cartesian Coordinates}

$$
\begin{aligned}
\nabla^{2} & =\frac{\partial^{2}}{\partial x^{2}}+\frac{\partial^{2}}{\partial y^{2}} \\
\nabla^{2} \nabla^{2} & =\frac{\partial^{4}}{\partial x^{4}}+2 \frac{\partial^{4}}{\partial x^{2} \partial y^{2}}+\frac{\partial^{4}}{\partial y^{4}}
\end{aligned}
$$

\section{B. Polar Coordinates}

$$
\begin{aligned}
\nabla^{2} & =\frac{1}{r} \frac{\partial}{\partial r}\left(r \frac{\partial}{\partial r}\right) \\
\nabla^{2} \nabla^{2} & =\left[\frac{1}{r} \frac{\partial}{\partial r}\left(r \frac{\partial}{\partial r}\right)\right]\left[\frac{1}{r} \frac{\partial}{\partial r}\left(r \frac{\partial}{\partial r}\right)\right]
\end{aligned}
$$

\section{APPENDIX B}

The calculated Galerkin solution for a circular plate is given by

$$
\begin{aligned}
V_{p i}^{(G)} & \\
= & {\left[\frac{26 D g^{3}}{\left(6237\left(169 \hat{\sigma}^{2}+3120 \hat{\sigma}+14400\right)\right) a^{4} \varepsilon}\right]^{1 / 2} } \\
& \times\left(67775488+18361728 \hat{\sigma}+1601424 \hat{\sigma}^{2}+44226 \hat{\sigma}^{3}\right. \\
& +\sqrt{2 / 13}[2749648671604736+1958606528643072 \hat{\sigma} \\
& +582159670456320 \hat{\sigma}^{2}+92420676311040 \hat{\sigma}^{3} \\
& +8265158066880 \hat{\sigma}^{4}+394789226832 \hat{\sigma}^{5} \\
& \left.\left.+7868724669 \hat{\sigma}^{6}\right]^{1 / 2}\right)^{1 / 2} \cdot
\end{aligned}
$$




\section{APPENDIX C}

A pressure difference across the plate will influence the pullin voltage, which easily can be calculated using the presented methodology. The governing equation for a circular CMUT then reads

$$
\nabla^{2} \nabla^{2} \hat{w}(\hat{r})-\hat{\sigma} \nabla^{2} \hat{w}(\hat{r})=\frac{\hat{\alpha}}{(1-\hat{w}(\hat{r}))^{2}}+\hat{p}
$$

where

$$
\hat{p}=\frac{\Delta p a^{4}}{D g}
$$

$\Delta p$ is the pressure difference across the plate. All other parameters are normalized by the definitions in table I. Substituting the static deflection profile into Eqn. (36) and equating the discriminant of this third order polynomial one can obtain the pull-in voltage given by

$$
V_{p i}^{(0)}=\frac{64}{9} \sqrt{\frac{2}{3} \frac{D g^{3}}{\varepsilon_{0} a^{4}} \frac{\left(1+\frac{\hat{\sigma}}{16}-\frac{9 \hat{p}}{1024}\right)^{3}}{\left(1+\frac{\hat{\sigma}}{16}\right)^{2}}} .
$$

The pressure reduces the initial gap, and the correction factor scales to the third power as the gap height, in perfect agreement with physical intuition. In addition when $\hat{p}=0$ the expression reduces to Eqn. (14), as expected.

\section{APPENDIX D}

By introducing the dimensionless parameters $\hat{w}=w / g$ and $\hat{x}=x / L$ the Euler-Bernoulli equation can be expressed as

$$
\frac{\mathrm{d}^{4} \hat{w}}{\mathrm{~d} \hat{x}^{4}}-\hat{N} \frac{\mathrm{d}^{2} \hat{w}}{\mathrm{~d} \hat{x}^{2}}=\hat{q},
$$

where $\hat{N}=\sigma_{0} W h L^{2} /(E I)$ and $\hat{q}=\varepsilon_{0} W V^{2} L^{4} /\left(2 g^{3}(1-\right.$ $w / g))$.

\section{REFERENCES}

[1] C.-H. Chu, W.-P. Shih, S.-Y. Chung, H.-C. Tsai, T.-K. Shing, and P.-Z. Chang, "A low actuation voltage electrostatic actuator for RF MEMS switch applications," J. Micromech. Microeng., vol. 17, no. 8, pp. 1649-1656, Aug. 2007.

[2] Y. Song, R. M. Panas, and J. B. Hopkins, "A review of micromirror arrays," Precis. Eng., vol. 51, pp. 729-761, Jan. 2018.

[3] K.-H. Jeong and L. P. Lee, "A novel microfabrication of a selfaligned vertical comb drive on a single SOI wafer for optical MEMS applications," J. Micromech. Microeng., vol. 15, no. 2, pp. 277-281, Feb. 2005.

[4] K. K. Park et al., "Capacitive micromachined ultrasonic transducer (CMUT) as a chemical sensor for DMMP detection," Sens. Actuators B, Chem., vol. 160, no. 1, pp. 1120-1127, Dec. 2011.

[5] A. S. Savoia, G. Caliano, and M. Pappalardo, "A CMUT probe for medical ultrasonography: From microfabrication to system integration," IEEE Trans. Ultrason., Ferroelectr., Freq. Control, vol. 59, no. 6, pp. 1127-1138, Jun. 2012.

[6] G. G. Yaralioglu, A. S. Ergun, B. Bayram, E. Haeggstrom, and B. T. Khuri-Yakub, "Calculation and measurement of electromechanical coupling coefficient of capacitive micromachined ultrasonic transducers," IEEE Trans. Ultrason., Ferroelectr., Freq. Control, vol. 50, no. 4, pp. 449-456, Apr. 2003.

[7] O. Oralkan et al., "Experimental characterization of collapse-mode CMUT operation," IEEE Trans. Ultrason., Ferroelectr., Freq. Control, vol. 53, no. 8, pp. 1513-1523, Aug. 2006.
[8] K. K. Park, O. Oralkan, and B. T. Khuri-Yakub, "A comparison between conventional and collapse-mode capacitive micromachined ultrasonic transducers in 10-MHz 1-D arrays," IEEE Trans. Ultrason., Ferroelectr., Freq. Control, vol. 60, no. 6, pp. 1245-1255, Jun. 2013.

[9] M. Pekař, W. U. Dittmer, N. Mihajlović, G. van Soest, and N. de Jong, "Frequency tuning of collapse-mode capacitive micromachined ultrasonic transducer," Ultrasonics, vol. 74, pp. 144-152, Feb. 2017.

[10] A. Nikoozadeh, B. Bayram, G. G. Yaralioglu, and B. T. Khuri-Yakub, "Analytical calculation of collapse voltage of CMUT membrane," in Proc. IEEE Ultrason. Symp., Apr. 2004, pp. 256-259.

[11] I. O. Wygant, M. Kupnik, and B. T. Khuri-Yakub, "Analytically calculating membrane displacement and the equivalent circuit model of a circular CMUT cell," in Proc. IEEE Ultrason. Symp., Nov. 2008, pp. 2111-2114.

[12] B. Ahmad and R. Pratap, "Elasto-electrostatic analysis of circular microplates used in capacitive micromachined ultrasonic transducers," IEEE Sensors J., vol. 10, no. 11, pp. 1767-1773, Nov. 2010.

[13] G. Duan and K.-T. Wan, "Pull-in' of a pre-stressed thin film by an electrostatic potential: A 1-D rectangular bridge and a 2-D circular diaphragm," Int. J. Mech. Sci., vol. 52, no. 9, pp. 1158-1166, 2010.

[14] M. F. la Cour, T. L. Christiansen, J. A. Jensen, and E. V. Thomsen, "Electrostatic and small-signal analysis of CMUTs with circular and square anisotropic plates," IEEE Trans. Ultrason., Ferroelectr., Freq. Control, vol. 62, no. 8, pp. 1563-1579, Aug. 2015.

[15] W. Zhang, H. Zhang, F. Du, J. Shi, S. Jin, and Z. Zeng, "Pull-in analysis of the flat circular CMUT cell featuring sealed cavity," Math. Problems Eng., vol. 2015, pp. 1-9, Sep. 2015.

[16] R. Maity, S. Shuvro, S. Maity, and N. P. Maity, "Collapse voltage analysis of central annular ring metallized membrane based MEMS micromachined ultrasonic transducer," Microsyst. Technol., vol. 26, no. 3, pp. 1001-1009, Mar. 2020.

[17] P. M. Osterberg and S. D. Senturia, "M-TEST: A test chip for MEMS material property measurement using electrostatically actuated test structures," J. Microelectromech. Syst., vol. 6, no. 2, pp. 107-118, Jun. 1997.

[18] M. I. Younis, E. M. Abdel-Rahman, and A. Nayfeh, "A reduced-order model for electrically actuated microbeam-based MEMS," J. Microelectromech. Syst., vol. 12, no. 5, pp. 672-680, Oct. 2003.

[19] G. W. Vogl and A. H. Nayfeh, "A reduced-order model for electrically actuated clamped circular plates," J. Micromech. Microeng., vol. 15, no. 4, p. 684,2005

[20] M. I. Haller and B. T. Khuri-Yakub, "A surface micromachined electrostatic ultrasonic air transducer," IEEE Trans. Ultrason., Ferroelectr., Freq. Control, vol. 43, no. 1, pp. 1-6, Jan. 1996.

[21] S. Timoshenko and S. Woinowsky-Krieger, Theory Plates Shells, 2nd ed. New York, NY, USA: McGraw-Hill, Jun. 1959.

[22] B. Wang, S. Zhou, J. Zhao, and X. Chen, "Pull-in instability analysis of electrostatically actuated microplate with rectangular shape," Int. J. Precis. Eng. Manuf., vol. 12, no. 6, pp. 1085-1094, Dec. 2011.

[23] M. Rahman, J. Hernandez, and S. Chowdhury, "An improved analytical method to design CMUTs with square diaphragms," IEEE Trans. Ultrason., Ferroelectr., Freq. Control, vol. 60, no. 4, pp. 834-845, Apr. 2013.

[24] R. Batra, M. Porfiri, and D. Spinello, "Effects of van der Waals force and thermal stresses on pull-in instability of clamped rectangular microplates," Sensors, vol. 8, no. 2, pp. 1048-1069, Feb. 2008.

[25] H. B. Chan, "Quantum mechanical actuation of microelectromechanical systems by the casimir force," Science, vol. 291, no. 5510, pp. 1941-1944, Mar. 2001.

[26] E. V. Thomsen, K. Reck, G. Skands, C. Bertelsen, and O. Hansen, "Silicon as an anisotropic mechanical material: Deflection of thin crystalline plates," Sens. Actuators A, Phys., vol. 220, pp. 347-364, Dec. 2014.

[27] A. S. Havreland, M. Engholm, C. V. Sorensen, and E. V. Thomsen, "Pullin analysis of CMUT elements," in Proc. IEEE Int. Ultrason. Symp. (IUS), Sep. 2020, pp. 1-4. 
[28] M. Engholm, T. Pedersen, and E. V. Thomsen, "Modeling of plates with multiple anisotropic layers and residual stress," Sens. Actuators A, Phys., vol. 240, pp. 70-79, Apr. 2016

[29] C. M. Bender and S. A. Orszag, Advanced Mathematical Methods for Scientists and Engineers. New York, NY, USA: McGraw-Hill, 1978.

[30] X. Zhao, E. M. Abdel-Rahman, and A. H. Nayfeh, "A reduced-order model for electrically actuated microplates," J. Micromech. Microeng., vol. 14, no. 7, p. 900, 2004.

[31] Z. Li et al., "Nonlinear behavior analysis of electrostatically actuated multilayer anisotropic microplates with residual stress," Compos. Struct., vol. 255, Jan. 2021, Art. no. 112964.

[32] A. Havreland, M. Ommen, C. Silvestre, M. Engholm, J. Jensen, and E. Thomsen, "BCB polymer based row-column addressed CMUT," in Proc. IEEE Int. Ultrason. Symp. (IUS), Sep. 2017, pp. $1-4$.

[33] A. Logan and J. T. W. Yeow, "Fabricating capacitive micromachined ultrasonic transducers with a novel siliconnitride-based wafer bonding process," IEEE Trans. Ultrason., Ferroelectr., Freq. Control, vol. 56, no. 5, pp. 1074-1084, May 2009.

[34] M. J. G. Mølgaard, M. Laustsen, M. H. Jakobsen, T. L. Andresen, and E. V. Thomsen, "Combined colorimetric and gravimetric CMUT sensor for detection of benzyl methyl ketone," Sens. Actuators B, Chem., vol. 275, pp. 483-489, Dec. 2018.

[35] K. S. Pister and S. B. Dong, "Elastic bending of layered plates," J. Eng. Mech. Division, vol. 85, no. 4, pp. 1-10, Oct. 1959.

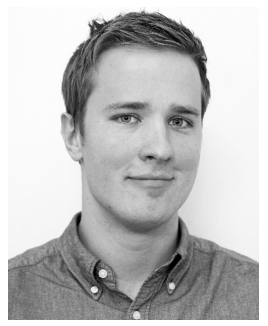

Andreas Spandet Havreland received the B.Sc. and M.Sc. degrees in engineering physics and nanotechnology and the Ph.D. degree with a focus on the development of capacitive micromachined ultrasonic transducers for 3-D imaging from the Technical University of Denmark, Kongens Lyngby, Denmark, in 2014, 2016, and 2020, respectively. $\mathrm{He}$ is currently working as a Development Engineer at Höttinger Brüel \& Kjær, where he is developing accelerometers.

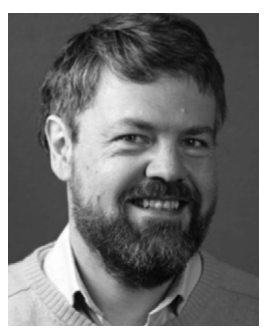

Erik Vilain Thomsen was born in Aarhus, Denmark, in 1964. He received the M.Sc. degree in physics from the University of Southern Denmark, Odense, Denmark, and the Ph.D. degree in electrical engineering from the Technical University of Denmark (DTU), Kongens Lyngby, Denmark, in 1998.

He is currently a Professor with DTU Nanotech, DTU, where he is also the Head of the MEMS Applied Sensors Group. He teaches classes in solidstate electronics, microtechnology, and nano and microfabrication. His current research interests include capacitive micromachined ultrasonic transducers, MEMS multisensors, biomedical devices, energy harvesting devices, and piezoelectric MEMS. He received the AEG Electron Prize in 1995. He also received several teaching awards at DTU. 